James Madison University JMU Scholarly Commons

2011

\title{
Heat recovery mechanism in the excitation of radiative polaritons by broadband infrared radiation in thin oxide films
}

\author{
Anita J. Vincent-Johnson \\ James Madison University \\ Kyle A. Vasquez \\ James Madison University \\ John E. Bridstrup \\ James Madison University \\ Andrew E. Masters \\ Custom Thermoelectric \\ Xiaofeng $\mathrm{Hu}$ \\ James Madison University \\ See next page for additional authors
}

Follow this and additional works at: http://commons.lib.jmu.edu/paa

Part of the Atomic, Molecular and Optical Physics Commons, Materials Chemistry Commons, and the Other Physics Commons

\section{Recommended Citation}

Vincent-Johnson, A. J., Vasquez, K. A., Bridstrup, J. E., Masters, A. E., Hu, X., Scarel, G. Heat recovery mechanism in the excitation of radiative polaritons by broadband infrared radiation in thin oxide films. Applied Physics Letters, 99, 131901 (2011),

DOI:http://dx.doi.org/10.1063/1.3643464 
Authors

Anita J. Vincent-Johnson, Kyle A. Vasquez, John E. Bridstrup, Andrew E. Masters, Xiaofeng Hu, and Giovanna Scarel 


\title{
Heat recovery mechanism in the excitation of radiative polaritons by broadband infrared radiation in thin oxide films
}

\author{
Anita J. Vincent-Johnson, ${ }^{1}$ Kyle A. Vasquez, ${ }^{2}$ John E. Bridstrup, ${ }^{1}$ Andrew E. Masters, ${ }^{3}$ \\ Xiaofeng $\mathrm{Hu}^{2}{ }^{2}$ and Giovanna Scarel ${ }^{1, \mathrm{a})}$ \\ ${ }^{1}$ Department of Physics and Astronomy, James Madison University, Harrisonburg, Virginia 22807, USA \\ ${ }^{2}$ Department of Chemistry and Biochemistry, James Madison University, Harrisonburg, Virginia 22807, USA \\ ${ }^{3}$ Custom Thermoelectric, Bishopville, Maryland 21813, USA
}

(Received 10 August 2011; accepted 1 September 2011; published online 26 September 2011)

\begin{abstract}
This work probes radiative polaritons in thin oxide layers as a mean to capture and absorb broadband infrared radiation and transform it into heat. A heat recovery mechanism, based on the Seebeck effect, is used as the tool of the investigation. Heat production challenges the current understanding which views the excitation of radiative polaritons as only accompanied by the emission of electromagnetic radiation. The heat recovery mechanism presented here can inspire the design of infrared energy harvesting devices, similar to photovoltaic cells, and other devices to convert energy from a wide range of the electromagnetic radiation spectrum using thermoelectric power generators. (C) 2011 American Institute of Physics. [doi:10.1063/1.3643464]
\end{abstract}

The objective of this work is to prove that (a) short lifetime $(\sim$ ps $)$ radiative polaritons $(\mathrm{RPs})^{1}$ excited by the coupling between transverse optical (TO) phonons in a thin oxide film and infrared (IR) photons produce heat and that (b) this heat can be transferred to a thermoelectric (TEC) power generator which, through a heat recovery mechanism based on the Seebeck effect, converts it into electricity. The theory by Kliewer et al. based on conservation of momentum $^{1,2}$ and on retardation ${ }^{1,2}$ predicts that the excitation of RPs is only followed by field radiation. For radiative plasmons in relatively ideal metal films, Ferrell proposed that radiation competes favorably with electronic damping and that the decay of the radiative plasmons manifests itself almost entirely as radiation. ${ }^{2}$ For RPs in dielectric layers, no phenomenon competing with radiation was predicted by Kliewer et al. ${ }^{1}$ This work indicates instead that heat production competes with radiation in the process of RP excitation, and that the produced heat is released within the thin oxide film.

A polariton is a mixed excitation resulting from the strong coupling between the TO phonons and the IR photon field in dielectric (oxide or semiconductor) layers. ${ }^{1}$ Differently than non-radiative polaritons, ${ }^{3}$ the RPs have a phase velocity such that $\left(\frac{\omega}{|\mathbf{k}|}\right)^{2}>(c)^{2},{ }^{1}$ where $\omega$ is the angular frequency, $|\mathbf{k}|$ is the modulus of the wave-vector, and $\mathrm{c}$ is the speed of light. Radiative polaritons respond to this unphysical situation by giving off energy. ${ }^{1}$ Generally, theory ${ }^{1}$ assumes that this energy is the radiation emitted at frequencies depending upon IR radiation incidence angle $\theta_{0}$ and oxide layer thickness. Therefore, the polaritons are named "radiative" and are held responsible of the optical properties in the IR region of the dielectric layers. ${ }^{4}$ Dissipation and damping phenomena, thus heat production, are not included in the theory of Kliewer et al., ${ }^{1,4}$ which only considers the boundary conditions between dielectric layers and perfect conductors or insulators. We thus hypothesizes that in thin oxide films on substrates with finite conductivity, heat is pro-

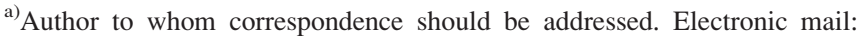
scarelgx@jmu.edu.
}

duced. We indirectly reveal the produced heat by the heat recovery mechanism as follows. Once produced in the excitation of RPs, heat can be transferred from the dielectric film to a TEC power generator. On the "hot" junction, the heat gives rise to a temperature difference $\Delta T$ with the "cold" junction. We experimentally estimated that the TEC power generator used in this work is sensitive down to $\Delta T \cong 2 * 10^{-3^{\circ}} \mathrm{C}$ for $2 * 10^{-4} \mathrm{~V}$. This $\Delta T$ is transformed into an electromotive force (EMF), i.e., electricity, via the Seebeck effect: $E M F=S \cdot \Delta T$, where $\mathrm{S}$ is the Seebeck coefficient. The IR radiation absorption by RPs has a marked dependence on the IR radiation incidence angle $\theta_{0}$ and polarization (transverse electric-TE or transverse magneticTM). ${ }^{1,4}$ Thus, to prove that the heat recovery mechanism is linked to RP excitation, here, we compare the dependence of the EMF versus $\theta_{0}$ with the dependence of the absorption of the RPs in the IR spectra versus $\theta_{0}$. The study with TE and TM polarized IR radiation will be pursued in a future experiment.

Amorphous (a) aluminium oxide $\left(\mathrm{Al}_{2} \mathrm{O}_{3}\right)$ films were deposited at $150^{\circ} \mathrm{C}$ on a $23 \mu \mathrm{m}$ thick $\mathrm{Al}$ foil via atomic layer deposition (ALD $)^{5}$ in a custom-made horizontal hot wall viscous flow reactor described elsewhere. ${ }^{6}$ The $\mathrm{Al}$ foil was cleaned by immersion in a $10 \% \mathrm{HF}$ solution for $60 \mathrm{~s}$. In each ALD cycle, the samples were exposed to $\mathrm{AlCH}_{3}$ and de-ionized $\mathrm{H}_{2} \mathrm{O}$ for $1 \mathrm{~s}$. Metal organic and water precursor pulses were followed by $8 \mathrm{~s}$ long purges in $\mathrm{Ar}$. The $\mathrm{aAl}_{2} \mathrm{O}_{3}$ film thickness on $\mathrm{H}$ terminated $\mathrm{Si}(100)$ was measured to be on average $250 \pm 30 \mathrm{~nm}$ using a variable-angle spectroscopic ellipsometer (J. A. Woollam Co.). The reflectance (R) of the $\mathrm{aAl}_{2} \mathrm{O}_{3}$ film on $\mathrm{Al}$ foil $\left(\mathrm{aAl}_{2} \mathrm{O}_{3} / \mathrm{Al}\right)$ was studied using a $\mathrm{N}_{2}$ purged Bruker Vertex 70 Fourier transform IR spectrometer in the $30-80^{\circ} \theta_{0}$ range. The broadband IR radiation is generated in the IR spectrometer by a globar (Q301) source. For the variable angle reflectance measurements, a Veemax II by Pike Technologies accessory was used. The IR radiation was polarized in the TE and TM states with a ZnSe middle-IR general purpose polarizer (Pike Technologies). The absorptance (A) spectra are plotted as $100 \%-\mathrm{R}$ versus frequency in $\mathrm{cm}^{-1}$ (Fig. 1). 


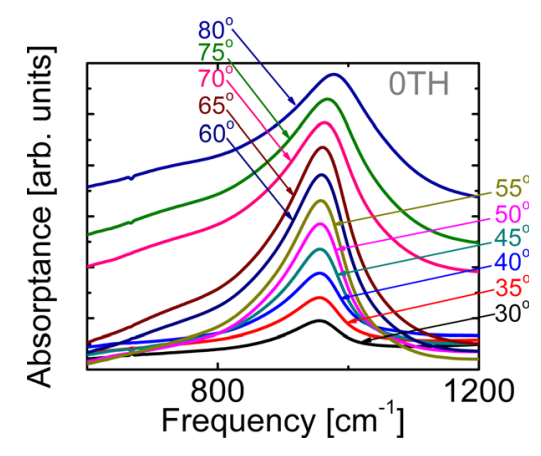

FIG. 1. (Color online) Absorptance of the 0TH type of RP in a $250 \mathrm{~nm}$ thick $\mathrm{aAl}_{2} \mathrm{O}_{3}$ film on $\mathrm{Al}$ foil illuminated by TM polarized broadband IR radiation at various values of $\theta_{0}$.

For the measurements involving the heat recovery mechanism, a bare and lapped TEC power generator with aluminaceramic surface (Custom Thermoelectric 07111-9L31-04B) was used. The TEC power generator has a nominal $0.14 \mathrm{~V} /{ }^{\circ} \mathrm{C}$ Seebeck coefficient, which is in good agreement with the experimental value of $0.097 \mathrm{~V} /{ }^{\circ} \mathrm{C}$. System 1 consists of a $250 \mathrm{~nm}$ thick $\mathrm{aAl}_{2} \mathrm{O}_{3}$ film on $\mathrm{Al}$ foil placed on a TEC power generator. System 2 consists of $\mathrm{Al}$ foil placed on a TEC power generator. The size of the $\mathrm{Al}$ and $\mathrm{aAl}_{2} \mathrm{O}_{3} / \mathrm{Al}$ foils was slightly smaller than the surface area of the "hot" junction of the TEC power generator. The $\mathrm{Al}$ and $\mathrm{aAl}_{2} \mathrm{O}_{3} / \mathrm{Al}$ foils were mounted on top of the "hot" junction of the TEC power generator and placed on the variable angle reflection accessory inside the sample compartment of the IR spectrometer with the IR source off. After connecting the leads of the TEC power generator with a Keithley 2000 multi-meter, sensitive to direct current (DC) voltages from $1 \mu \mathrm{V}$ to $1 \mathrm{kV}$, the sample compartment was closed and left in the dark laboratory room at $20{ }^{\circ} \mathrm{C}$. A reading of $0.01 \mathrm{mV}$ on the multi-meter indicated that the systems had achieved a stable initial condition for the experiment. Systems 1 and 2, illustrated in the inset of Fig. 2, were then exposed to the broadband non-polarized IR radiation in the middle IR region $\left(350-4000 \mathrm{~cm}^{-1}\right)$ generated by the Q301globar source in the IR spectrometer. As soon as the systems were illuminated by the IR radiation at a certain

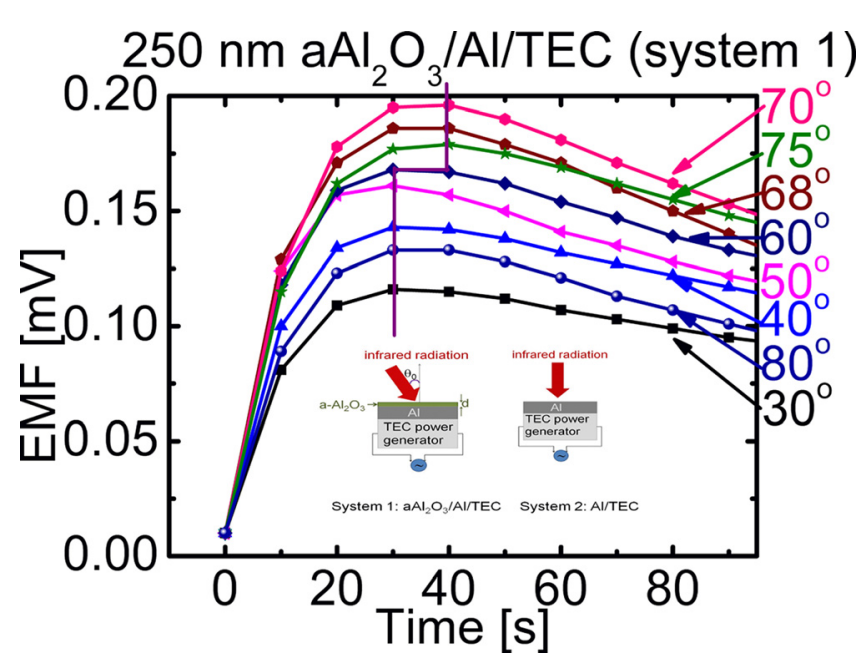

FIG. 2. (Color online). Time evolution of the EMF generated upon illuminating system 1 at selected values of $\theta_{0}$. The vertical lines mark the $\mathrm{EMF}_{\max }$ values. Inset: Schematics of systems 1 and 2 . angle $\theta_{0}$ from $30^{\circ}$ to $80^{\circ}$, an EMF was generated. The relaxation of the EMF was then observed for $6 \mathrm{~min}$ and $40 \mathrm{~s}$ after turning on the broadband IR radiation. In that time interval, the EMF values were collected every $10 \mathrm{~s}$ during the first minute and $40 \mathrm{~s}$, and then, in the following $5 \mathrm{~min}$, every $20 \mathrm{~s}$.

Figure 1 depicts the absorptance spectra of the $250 \pm 30 \mathrm{~nm}$ thick $\mathrm{aAl}_{2} \mathrm{O}_{3}$ film on the $\mathrm{Al}$ foil. The spectra were collected with TM polarized broadband IR radiation in the $30-80^{\circ} \quad \theta_{0}$ range. The only peak appears around $950 \mathrm{~cm}^{-1}$, slightly blue shifting with increasing $\theta_{0}$. The peak corresponds to the 0TH type of RP excited near the longitudinal optical (LO) phonon $\mathrm{LO} 4$ frequency of $\mathrm{aAl}_{2} \mathrm{O}_{3} \cdot{ }^{1,7}$ Its absorptance increases with $\theta_{0}$, a phenomenon known as the Berreman effect. ${ }^{8}$ For the same sample, RPs are absent in spectra collected with TE polarized broadband IR radiation in the middle IR region.

Figure 2 shows the time relaxation of the EMF in a TEC power generator after the excitation of RPs in system 1 with broadband middle-IR radiation at selected values of $\theta_{0}$. It can be observed that (1) the maxima of the EMF values $\left(\mathrm{EMF}_{\max }\right)$ depend on $\theta_{0}$ and (2) the $\mathrm{EMF}_{\text {max }}$ values are achieved with a $40 \mathrm{~s}$ delay for $\theta_{0}>70^{\circ}$ and with a $30 \mathrm{~s}$ delay for $\theta_{0}<70^{\circ}$. Similar delays were found for system 2 . This response is constrained by the intrinsic time constant of the TEC power generator. After exciting the RPs and reaching the $\mathrm{EMF}_{\max }$ value, systems 1 and 2 relax quasi-exponentially for a certain time interval, while the IR radiation keeps illuminating them. We believe that, in accordance with our hypothesis, the quasi-exponential relaxation is linked to the limited lifetime (few ps) of the RPs and retarded by the intrinsic time constant of the TEC power generator. To formulate a theoretical model, the results of further experimental studies are needed, in particular those performed with TE and TM polarized IR radiation.

Figure 3(a) displays the $\mathrm{EMF}_{\text {max }}$ values versus $\theta_{0}$ for systems 1 (filled triangles) and 2 (filled circles). The error is the standard deviation obtained from two measurements for each point. The $\mathrm{EMF}_{\max }$ values of system 1 are always higher than those of system 2 . The actual difference is reported versus $\theta_{0}$ in Fig. 3(b), where, at each angle, the system providing the (a)

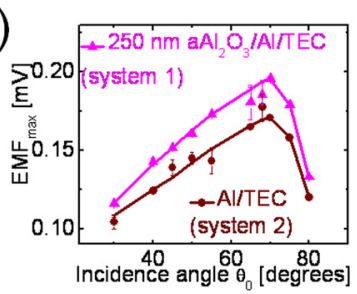

(c)

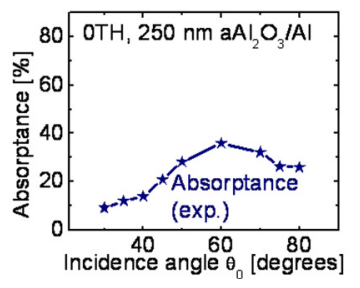

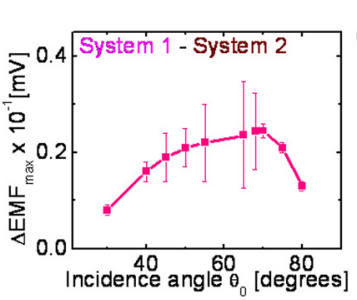

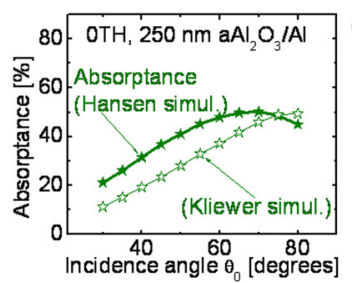

FIG. 3. (Color online) (a) $\mathrm{EMF}_{\max }$ versus $\theta_{0}$ for system 1 (filled triangles) and system 2 (filled circles). (b) Difference between $\mathrm{EMF}_{\max }$ versus $\theta_{0}$ for systems 1 and 2. Experimental (c) and simulated (d) absorptance versus $\theta_{0}$ of the 0TH type of RP from IR spectra. The simulations were obtained from the Hansen (Ref. 9) and Kliewer (Ref. 4) methods. 
larger error determines the uncertainty. The difference in $\mathrm{EMF}_{\max }$ values between systems 1 and 2 increases with $\theta_{0}$ from $30^{\circ}$ to $\sim 70^{\circ}$ and decreases from $\sim 70^{\circ}$ to $80^{\circ}$. This trend resembles that of the absorptance versus $\theta_{0}$ of the $0 \mathrm{TH}$ type of $\mathrm{RP}$ in a $250 \mathrm{~nm}$ thick $\mathrm{aAl}_{2} \mathrm{O}_{3}$ film on $\mathrm{Al}$ foil derived in Fig. 3(c) from the experimental data in Fig. 1 and in Fig. 3(d) from simulations obtained with the Hansen method. ${ }^{9}$

Figure 3(d) also compares the absorptance versus $\theta_{0}$ of the 0TH type of RP in a $250 \mathrm{~nm}$ thick $\mathrm{aAl}_{2} \mathrm{O}_{3}$ film on $\mathrm{Al}$ foil derived from the Hansen ${ }^{9}$ and Kliewer ${ }^{4}$ methods. The two methods differ in that the former assumes a metal layer with the finite conductivity of $\mathrm{Al}$, while the latter assumes infinite conductivity. The absorptance derived from the Kliewer method $^{4}$ is solely due to the $0 \mathrm{TH}$ type of RP, is lower than that obtained with the Hansen method, and does not exhibit a sharp maximum at $70^{\circ}$.

The correlation found here between the difference in EMF $_{\text {max }}$ of systems 1 and 2 in Fig. 3(b), and the absorptance versus $\theta_{0}$ of the $0 \mathrm{TH}$ type of $\mathrm{RP}$ in a $250 \mathrm{~nm}$ thick $\mathrm{aAl}_{2} \mathrm{O}_{3}$ film on Al foil in Figs. 3(c) and 3(d), supports the hypothesis that heat (i) is produced in the excitation of the 0TH type of RPs and (ii) can be revealed through the recovery mechanism. As shown in Fig. 3(d), this phenomenon was not predicted in the RPs theory of Kliewer et al., ${ }^{1}$ where all energy was confined by laws of conservation of momentum and retardation in the radiated fields. Considering that a substrate with finite conductivity was used in the Hansen method, and that chemistry and conductivity of the substrate profoundly influence the IR radiation absorption by RP excitation, as inferred from Fig. 3(d), we argue that dissipation phenomena take place, and that the energy from the incident broadband IR radiation can be converted in the thin oxide films into something other than radiative energy.

The large uncertainties in Fig. 3(b) are attributed to the continuous illumination of systems 1 and 2 by IR radiation during the EMF relaxation. These uncertainties blur the response to the RP excitation of the TEC power generator constrained by its time constant. Exploring the heat recovery mechanism by exciting the RPs with stable ultra-fast and high repetition rate photon pulses of the order of magnitude of the RP lifetime, or even less, should lower the uncertainties in the determination of the $\mathrm{EMF}_{\max }$ values. This experiment is planned in the near future and is expected to unambiguously link the excitation of RPs in IR radiation absorption to the $\mathrm{EMF}_{\max }$ values.

In summary, a correlation is found between the angular dependence of the heat recovery mechanism and the absorptance of the 0TH type of RPs excited in thin alumina films. This result indicates that heat is produced in the excitation of RPs. To unambiguously prove this correlation, stable, ultrafast, and high repetition rate photon pulses of the order of magnitude of the RP lifetime should be used. These results are expected to impact the exploitation of RPs to convert IR radiation into usable energy, and could inspire the future exploration, for energy conversion purposes, of the interaction between TEC power generators and radiation in a broad spectrum of frequencies including the visible, ultraviolet, and $\mathrm{X}$-ray regions.

The JMU Center for Materials Science, JMU Department of Physics and Astronomy, NSF-REU and Department of Defense ASSURE program (Grant No. 0851367), Research Corporation Science Department Development Grant 7957, and Summer Research Grant 2011-JMU College of Science and Mathematics, are acknowledged for funding. Professor Dorn W. Peterson (JMU), and Dr. Gwyn P. Williams (Thomas Jefferson National Accelerator Facility, Newport News, VA) are acknowledged for fruitful discussions.

${ }^{1}$ K. L. Kliewer and R. Fuchs, Phys. Rev. 150, 573 (1966).

${ }^{2}$ R. A. Ferrell, Phys. Rev. 111, 1214 (1958).

${ }^{3}$ K. L. Kliewer and R. Fuchs, Phys. Rev. 144, 495 (1966).

${ }^{4}$ R. Fuchs, K. L. Kliewer, and W. J. Pardee, Phys. Rev. 150, 589 (1966).

${ }^{5} \mathrm{M}$. Ritala and M. Leskelä, in Handbook of thin film materials, edited by H. S. Nalwa (Academic, San Diego, CA, 2002), Vol. 1, pp. 103-159.

${ }^{6}$ K. A. Vasquez, A. J. Vincent-Johnson, W. C. Hughes, B. H. Augustine, K. Lee, G. N. Parsons, and G. Scarel, "Wetting properties induced in nano-composite POSS-MA polymer films by atomic layer deposited oxides," J. Vac. Sci. Technol. A (to be published).

${ }^{7}$ Y. T. Chu, J. B. Bates, C. W. White, and G. C. Farlow, J. Appl. Phys. 64, 3727 (1988).

${ }^{8}$ D. W. Berreman, Phys. Rev. 130, 2193 (1963).

${ }^{9}$ W. N. Hansen, J. Opt. Soc. Am. 58, 380 (1968). 